\title{
Escherichia coli Modulator of Drug Activity B (MdaB) has different enzymological properties to eukaryote quinone oxidoreducatases
}

\author{
Clare F. Megarity and David J. Timson. ${ }^{\mathrm{a}, \mathrm{b}, *}$ \\ a School of Biological Sciences, Queen's University Belfast, Medical Biology Centre, 97 Lisburn Road, Belfast, BT9 7BL. UK

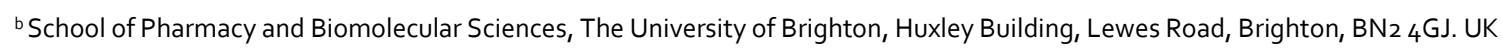 \\ d.timson@brighton.ac.uk
}

\begin{abstract}
Some quinone oxidoreductases exhibit negative cooperativity towards inhibitors. In human $\mathrm{NOO}_{1}$, this is mediated by flexibility around glycine-150. Here we investigated the eubacterial orthologue, Modulator of Drug Activity B (MdaB) to determine if it shows cooperativty towards substrates or inhibitors and to investigate molecular recognition of the inhibitor, dicoumarol. Like human $\mathrm{NOO}_{1}, \mathrm{MdaB}$ did not show cooperativity towards substrates. However, unlike $\mathrm{NOO}_{1}$, it was only weakly inhibited by dicoumarol. Alanine-127 in MdaB is the structurally equivalent residue to Gly150 in human NOO1. With the intention of increasing protein flexibility in MdaB, this alanine was altered to glycine. This change did not increase cooperativity towards inhibitors or NADPH. Based on structural alignment to $\mathrm{NOO}_{1}$ in complex with dicoumarol, an asparagine in the active site was changed to alanine to reduce steric hindrance. This change resulted in enhanced inhibition by dicoumarol, but the inhibition was not cooperative. Both changes were then introduced simultaneously. However, the additional increase in flexibility afforded by the change to glycine did not enable negative cooperativity towards dicoumarol. These results have implications for the evolution of quinone oxidoreductases and their potential use as biocatalytsts.
\end{abstract}

Keywords: quinone oxidoreductase $\bullet$ dicoumarol $\bullet$ protein engineering $\bullet$ cooperativity $\bullet$ enzyme catalysis

\section{Introduction}

Quinone oxidoreductases catalyse the two electron reduction of quinones at the expense of NADH or NADPH. The best characterized example is mammalian $\mathrm{NAD}(\mathrm{P}) \mathrm{H}$ quinone oxidoreductase 1 ( $\mathrm{NOO}_{1}$, DT-diaphorase, $E C$ 1.6.5.2 ${ }^{[1-3]}$. This enzyme has received considerable attention due to its potential as a cancer chemotherapy target, its postulated role in vitamin $\mathrm{K}$ metabolism and its interactions with cell signaling molecules such as p53 [4]. Considerably less is known about quinone oxidoreductases from eubacteria. However, organisms from this domain often express several enzymes in this class. These include the nitroreductase enzymes (e.g. NemA, NfsA, NfsB, YdjA) and NOO1-like enzymes such as MdaB ${ }^{[5-10]}$. $M d a B$ reduces quinones by the transfer of two electrons; therefore like $\mathrm{NOO}_{1}$ it has a postulated role in their detoxification. In Escherichia coli, the expression of $\mathrm{MdaB}$ is increased in the presence of menadione ${ }^{[11]}$. MdaB is proposed to work in tandem with a quinol monoxygenase (QuMo), forming a quinone redox cycle which enables $E$. coli to maintain an intracellular store of menadione (required for anaerobic growth) without the formation of semiquinones ${ }^{[2]}$. MdaB confers resistance to the antibiotics adriamycin, etoposide and DMP840 while QuMo confers resistance to adriamycin and tetracycline; these molecules are structurally similar to napthoquinones which point to a role in the cycling of these molecules until they can be conjugated ${ }^{[1]]}$. There is also industrial interest in broad specificity oxidoreductase enzymes ${ }^{\left[{ }^{[3]}\right]}$. MdaB has potential since it is relatively stable and has an accommodating active site.

Mammalian $\mathrm{NOO}_{1}$ and bacterial MdaB both have a tightly bound FAD cofactor ${ }^{[11,14]}$; in contrast the nitroreductases and Lot6p proteins from budding yeasts use FMN [7-9, 15-17]. In both groups, the flavin cofactor plays a key role in catalysis, accepting electrons from NADH or NADPH in the first step of the reaction. The oxidised $N A D(P)^{+}$then exits the active site and is replaced by the second substrate which is then reduced by the flavin cofactor ${ }^{[14]}$. MdaB reduces quinones by the transfer of two electrons suggesting that it is involved in their detoxification ${ }^{[18]}$. MdaB is a dimer with a classic flavodoxin fold. Each monomer has a large catalytic domain which aligns well with human and rat $\mathrm{NOO}_{1}{ }^{[1]}$.

Human $\mathrm{NAD}(\mathrm{P}) \mathrm{H}$ quinone oxidoreductase 1 ( $\mathrm{NOO} 1$ ), human $\mathrm{NRH}$ quinone oxidoreductase $2\left(\mathrm{NOO}_{2}\right)$ and budding yeast Lot6p all exhibit negative cooperativity towards inhibitors such as dicoumarol and resveratrol [19-22]. Negative cooperativity is a decrease in affinity when sequential molecules of ligand bind ${ }^{[23,24]}$. Binding of the first molecule causes a conformational change which is propagated through the protein and causes the effect elsewhere. Cooperativity, like catalysis, is therefore inextricably linked to protein motion and flexibility ${ }^{[24]}$. In human $\mathrm{NOO}_{1}$, glycine 150 has been identified as pivotal to the mechanism and a change from glycine to the more conformationally restricting residue serine at this position, largely abolished negative cooperativity ${ }^{[25]}$.

$\mathrm{MdaB}$ was structurally aligned to human $\mathrm{NOO}_{1}\left(\mathrm{PDB}_{2} \mathrm{~F}_{1} \mathrm{O}^{[26]}\right.$ ) and the equivalent residue to the pivotal glycine in $\mathrm{NOO}_{1}$ was identified as Ala-127 in MdaB. To investigate whether, or not, negative cooperativity towards inhibitors could be introduced into MdaB, this alanine was changed to glycine in order to increase conformational freedom and flexibility at this position ${ }^{[27]}$. Dicoumarol inhibits $\mathrm{NOO}_{1}$ by binding in its active site; it $\pi$ stacks with the isoalloxazine ring system of the FAD cofactor ${ }^{[26]}$. Since dicoumarol is a poor inhibitor of the oxidoreductase activity of MdaB, structural alignment with $\mathrm{NOO}_{1}$ in complex with dicoumarol (PDB: $2 \mathrm{~F}_{1} \mathrm{O}$ ${ }^{[26]}$ ) was used to determine possible reasons why dicoumarol did not 


\section{HELVETICA}

favourably bind in the active site of MdaB. The alignment showed an asparagine residue in $\mathrm{MdaB}$ which may sterically hinder dicoumarol by protrusion into its potential binding pocket, preventing its optimum orientation. In particular, it was predicted to disrupt the potential for $\pi$ stacking with FAD (Fig. 1). To reduce this steric hindrance, asparagine was changed to alanine with a view to facilitate dicoumarol binding and therefore enhance its inhibition of MdaB.

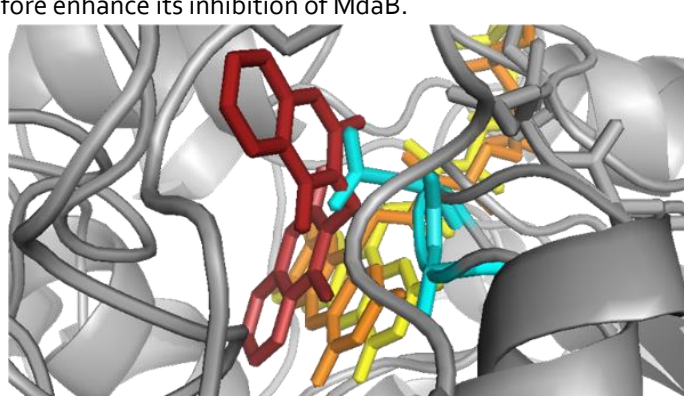

Figure 1. Alignment of human $\mathrm{NQO}_{1}\left(\mathrm{PDB}_{2} \mathrm{~F}_{1} \mathrm{O}^{[26]}\right.$ ) with $\mathrm{MdaB}$ (PDB $2 \mathrm{~B}_{3} \mathrm{D}^{[11]}$ ) showing the protrusion of asparagine 126 (cyan) in $\mathrm{MdaB}$ into the dicoumarol (red) binding site in $\mathrm{NQO}_{1}$. The position of the FAD cofactor in $\mathrm{NOO}_{1}$ and $\mathrm{MdaB}$ are shown in yellow and orange respectively.

\section{Results and Discussion}

The wild-type and variant forms of $\mathrm{MdaB}$ could be expressed in, and purified from, E. coli and, as expected, all dimerised as indicted by chemical crosslinking (data not shown).

Wild-type MdaB exhibited monophasic thermal denaturation with a "melting temperature" $\left(T_{m}\right)$ of $61.8 \pm 0.5^{\circ} \mathrm{C}$. All variant enzymes also exhibited monophasic melting (Supplementary Fig. S1). The change from alanine to glycine at position 127 destabilised the enzyme while the change from asparagine to alanine at position 126 was stabilising $\left(T_{m}=53.4 \pm 0.1{ }^{\circ} \mathrm{C}\right.$ and $63.5 \pm 0.0^{\circ} \mathrm{C}$ respectively). The simultaneous change at both positions was, overall, destabilising, but to a lesser extent than the destabilising effect of the single change from alanine to glycine $\left(T_{m}=56.5 \pm 0.0^{\circ} \mathrm{C}\right.$; Supplementary Fig. $\left.\mathrm{S}_{1}\right)$. Residue 127 , while close to the enzyme's surface, is mostly buried. Examination of a space-filling model shows that it is likely to have access to solvent (data not shown). The effect of glycine relative to alanine for a solvent-exposed internal residue is likely to be destabilising because (i) the amount of buried hydrophobic surface area is less, (ii) it makes fewer van der Waals contacts and (iii) it loses more entropy on folding ${ }^{[28]}$. Residue 126 is positioned in a loop just before a small helix, protruding into the active site close to FAD and is partially solvent exposed. Asparagine relative to alanine at this position is likely to be destabilising as it may hydrogen bond to the backbone in an unfolded states and there would be a reduction in conformational freedom and of the solvation of the asparagine. The destabilisation caused by Ala127 to Gly was presumably greater than the stability afforded by the change form Asn-126 to Ala explaining a net destabilisation of the double variant.
Wild-type MdaB was active with NADPH as an electron donor and DCPIP as an electron acceptor (Fig 2); there was no detectable activity with $\mathrm{NADH}$ as electron donor (data not shown). Variants, p.A127G, p.N126A and p. N126A/A127G are active with NADPH as an electron donor and DCPIP as an electron acceptor. The $k_{\mathrm{cat}, \mathrm{app}} / K_{\mathrm{m}, \mathrm{app}}$ value for $\mathrm{p} . \mathrm{A} 127 \mathrm{G}$ was greater than that for the wild-type enzyme (Table 1; Fig. 2) indicating that the change from alanine to glycine caused an increase in the enzyme's activity with these substrates. The $k_{c a t, a p p} / K_{m, a p p}$ values for the p.N126A and p.N126A/A127G variants were lower than that for the wild-type enzyme (Table 1; Fig. 2). Wild-type MdaB does not exhibit negative cooperativity towards NADPH $h=1.05 \pm 0.03$. None of the changes to the enzyme affected cooperativity towards the electron donor as the values of $h$ for each variant enzyme were close to 1 (Table 1 ; Fig. 2).

The ligands nicotinamide, NADP ${ }^{+}$and dicoumarol stabilised the WT enzyme towards thermal denaturation and this stabilisation was concentration-dependent (Fig. 3; Supplementary Fig. S2; Table 1). Hyperbolic behaviour was observed for nicotinamide but not for dicoumarol or for $\mathrm{NADP}^{+}$at the maximum concentrations possible under the experimental conditions used. The curve for dicoumarol was almost linear. This indicates that the binding of dicoumarol is not exclusively in the active site of the WT enzyme or that the concentration range used was much lower than the $K_{\mathrm{D} \text {, app }}$ value.

The wild-type oxidoreductase activity was inhibited in a concentrationdependent manner by nicotinamide and NADP+; dicoumarol $(100 \mu \mathrm{M})$ did not inhibit the oxidoreductase activity. Previously it was reported the inhibition of a bacterial oxidoreductase by dicoumarol with a competitive inhibition constant of $0.22 \mu \mathrm{M}^{[29]}$. However, the authors reported the cofactor of this oxidoreductase to be FMN; the crystal structure of MdaB shows its cofactor to be FAD ${ }^{[11]}$. Therefore, although the authors drew a parallel between this enzyme and rat $\mathrm{NQO}_{1}$ it is not likely to be MdaB. The type of inhibition observed was mixed for $\mathrm{NADP}^{+}$and nicotinamide.

Negative cooperativity was observed towards NADP ${ }^{+}, h=0.74 \pm 0.07$; slight positive cooperativity was observed towards nicotinamide, $h=1.20 \pm 0.12$ (Table 1; Fig. 3). The occurrence of both positive and negative cooperativity towards different ligands has been observed in other enzymes such as CTP synthetase and glyceraldehyde 3-phosphate dehydrogenase ${ }^{\left[3^{0}, 3^{13}\right]}$. Nicotinamide is a structural component of NADPH and $\mathrm{NADP}^{+}$yet it induces slight positive cooperativity. The contrasting behaviour of $\mathrm{NADP}^{+}$and NADPH is the most surprising since their only difference is that NADPH is the reduced form. The nature of the ping-pong mechanism provides an explanation; NADPH binds in the active site and donates two electrons to FAD and leaves the active site as the oxidised form. MdaB therefore undergoes conformational changes to facilitate the binding and release of the electron donor and since the binding of NADP+ is overall unfavourable as it is released from the active site, this infers that there are subtle differences in its binding mode compared to that for $\mathrm{NADPH}$. These would allow $\mathrm{NADP}^{+}$to induce negative cooperativity through a different binding mode than that of NADPH which does not induce cooperativity. The cooperativity towards $\mathrm{NADP}^{+}$and nicotinamide 


\section{HELVETICA}

could also result from the effects of binding to different sites on the enzyme as indicated by mixed inhibition.

The p.A127G variant was also stabilised towards thermal denaturation by dicoumarol and the relationship was saturating. Nicotinamide destabilised this variant and $\mathrm{NADP}^{+}$had no effect (Table 1; Fig. 3; Supplementary Fig.

S2). Favourable binding of a ligand to a partially unfolded protein can manifest as a destabilisation of the protein [32].

The oxidoreductase activity of the p.A127 G variant was also inhibited by these ligands in a concentration-dependent manner (Table 1; Fig. 2). The change from alanine to glycine did not affect the cooperativity towards $\mathrm{NADP}^{+}$. There was however, a slight increase in the value of $h$ for nicotinamide to $1.40 \pm 0.12$ (Table 1; Fig. 2). This indicates that alanine at position 127 may be involved in the cooperative effects induced by nicotinamide. It is likely that the alanine residue either contacts the nicotinamide directly or contacts the residues which interact first with the ligand. Moreover, since it is positioned in a loop at the end of a helical arrangement linking the two active sites, it is feasible that it is involved in the communication between them. This could occur by large scale backbone motion of the pathway and/or conformational changes of its residues and/or changes in dynamics.

The p.N126A variant was stabilised towards thermal denaturation by dicoumarol (Table 1; Fig. 3; Supplementary Fig. S2); the $K_{\mathrm{D} \text {, app }}$ is considerably less than that for wild-type, suggesting tighter binding. Surprisingly, p.A127G bound dicoumarol most tightly (Table 1; Fig. 3). On binding, the favourable interactions such as hydrogen bonds and van der Waal's interactions between dicoumarol and p.A127G combined with any increase in entropy which occur (for example, in the solvent) must outweigh the unfavourable decrease in entropy for both p.A127G and dicoumarol. The overall Gibb's free energy for dicoumarol binding to p.A127G must be more negative than that for dicoumarol binding to wildtype and, surprisingly, p.N126A. Since dicoumarol was shown to be a poor inhibitor of the oxidoreductase activity of p.A127G, it can be assumed that the tight binding does not interfere directly with substrate binding and occur away from the active site.

In the presence of $100 \mu \mathrm{M}$ dicoumarol, wild-type and $\mathrm{p} . \mathrm{A} 127 \mathrm{G}$ retained most of their activity and p.N126A became almost inactive

(Supplementary fig. S3). Dixon plot analysis showed that the inhibition is competitive with respect to NADPH (Data not shown). The change from asparagine to alanine allowed enhanced inhibition by dicoumarol, most likely by reduction of steric hindrance in the active site. Unlike $\mathrm{NQO}_{1}$, negative cooperativity towards dicoumarol was not observed in this dicoumarol accommodating variant ( $h=0.93 \pm 0.02$; Table 1; Fig. 2). Both the dicoumarol-accommodating change and the change to increase flexibility were introduced simultaneously with the intention of investigating the effect on cooperativity towards dicoumarol. Dicoumarol also stabilised the p.N126A/A127G variant towards thermal denaturation with a slightly lower $K_{\mathrm{D} \text {,app }}$ value than that for the p.N126A variant. In contrast, p.N126A/A127G has a much higher $K_{i, \text { app }}$ value for dicoumarol than p.N126A (Table 1); therefore at a given concentration of dicoumarol more molecules of p.N126A are in complex with dicoumarol compared with p.N126A/A127G. $K_{\mathrm{D} \text {, app }}$ values for dicoumarol binding to these two variants do not reflect this (Table 1 ); the $K_{\mathrm{i}, \text { app }}$ values are obtained kinetically and show that the inhibition of the double mutant is mixed while for p.N126A it is competitive. As expected, inhibition by dicoumarol occurs. However, the additional change to glycine has changed its nature from competitive to mixed (data not shown). Since this residue is in the active site, it is most likely that this change to glycine has increased the plasticity of the active site and allowed dicoumarol binding in different orientations. The Hill coefficient for dicoumarol is to the same as that for p.N126A. Therefore the increase in flexibility afforded by the additional change to glycine did not enable MdaB to exhibit negative cooperativity towards dicoumarol (Table 1; Fig. 2).

Table 1 Kinetic and apparent binding constants for WT MdaB and variants

\begin{tabular}{|c|c|c|c|c|}
\hline Inhibitor & WT & p.A127G & p.N126A & $\begin{array}{l}\text { p.N126A/A12 } \\
{ }_{7} \mathrm{G}\end{array}$ \\
\hline \multirow{3}{*}{ None } & \multicolumn{4}{|c|}{$k_{\mathrm{cat}} / K_{\mathrm{m}} / \mu \mathrm{M}^{-1} \mathbf{s}^{-1}$} \\
\hline & $0.23 \pm 0.02$ & $1.00 \pm 0.17$ & $\begin{array}{l}0.007 \pm 0.00 \\
1\end{array}$ & $0.018 \pm 0.003$ \\
\hline & \multicolumn{4}{|c|}{$K_{\text {ic,app }} / \mathrm{mM}$} \\
\hline Dicoumarol & - & - & $2.8 \pm 1.2$ & $23.8 \pm 7.0$ \\
\hline $\begin{array}{c}\text { Nicotinamid } \\
e\end{array}$ & $218 \pm 4$ & Indeterminable & - & - \\
\hline $\mathrm{NADP}^{+}$ & $0.7 \pm 0.4$ & $2.3 \pm 0.0$ & - & - \\
\hline
\end{tabular}

\begin{tabular}{ccccc}
\hline & \multicolumn{4}{c}{$K_{\text {iu,app } / \mathbf{m M}}$} \\
\cline { 2 - 5 } $\begin{array}{c}\text { Nicotinamid } \\
\mathrm{e}\end{array}$ & $1369 \pm 476$ & - & - & - \\
$\mathrm{NADP}^{+}$ & $14.6 \pm 6.2$ & $14.3 \pm 6.4$ & - & -
\end{tabular}

\begin{tabular}{|c|c|c|c|c|}
\hline \multirow[b]{2}{*}{ Dicoumarol } & \multicolumn{4}{|c|}{ Hill Coefficient $(h)$} \\
\hline & - & - & $0.93 \pm 0.02$ & $0.93 \pm 0.02$ \\
\hline $\begin{array}{c}\text { Nicotinamid } \\
\mathrm{e}\end{array}$ & $1.20 \pm 0.12$ & $1.40 \pm 0.12$ & - & - \\
\hline $\mathrm{NADP}^{+}$ & $0.74 \pm 0.07$ & $0.71 \pm 0.05$ & - & - \\
\hline \multirow[t]{2}{*}{ NADPH } & $1.05 \pm 0.03$ & $1.01 \pm 0.20$ & $0.92 \pm 0.04$ & $0.92 \pm 0.03$ \\
\hline & \multicolumn{4}{|c|}{$K_{\mathrm{D}, \mathrm{app}} / \mathrm{mM}$} \\
\hline Dicoumarol & $\begin{array}{c}0.526 \pm 0.2 \\
07\end{array}$ & $\begin{array}{c}0.048 \pm 0.00 \\
7\end{array}$ & $\begin{array}{c}0.151 \pm 0.03 \\
1\end{array}$ & $0.120 \pm 0.030$ \\
\hline $\begin{array}{c}\text { Nicotinamid } \\
\mathrm{e}\end{array}$ & $24 \pm 12$ & Destabilised & - & - \\
\hline $\mathrm{NADP}^{+}$ & $8 \pm 1$ & No effect & - & - \\
\hline
\end{tabular}




\section{HELVETICA}

WT

A
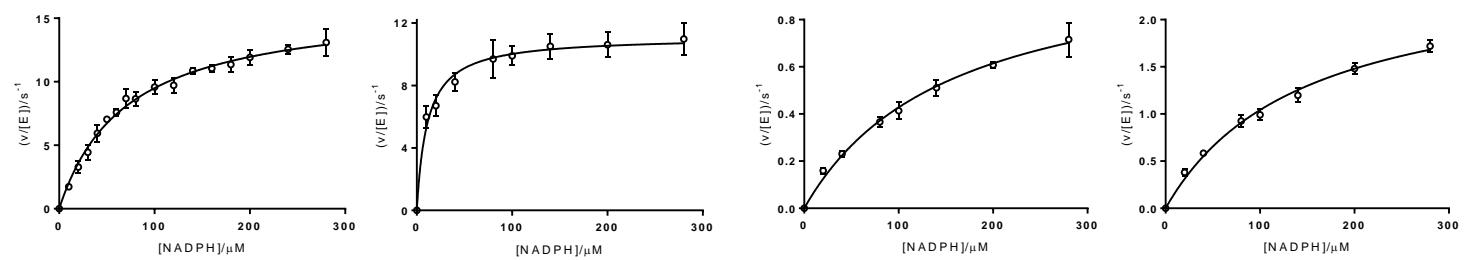

B
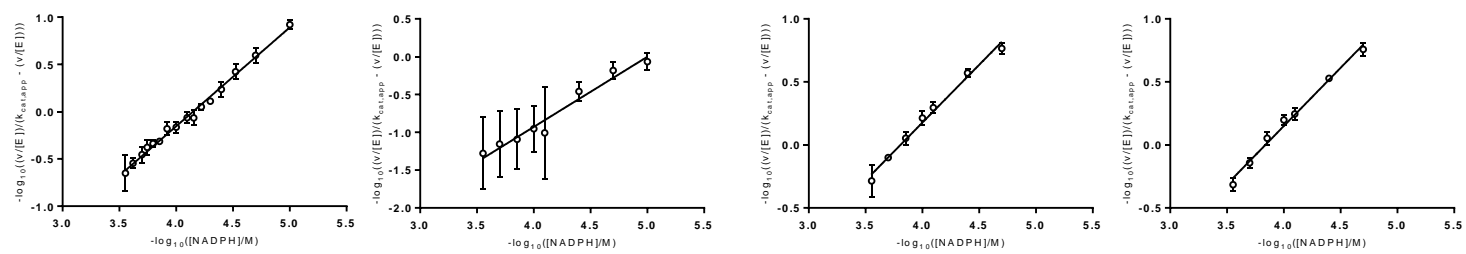

C
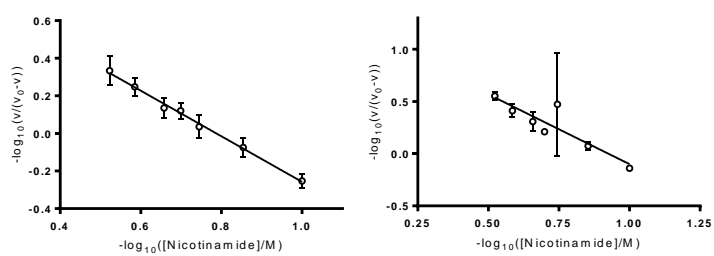

D
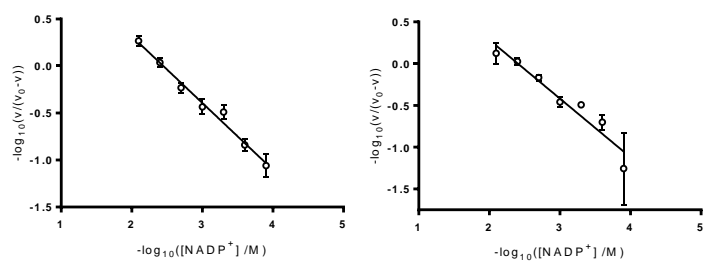

E

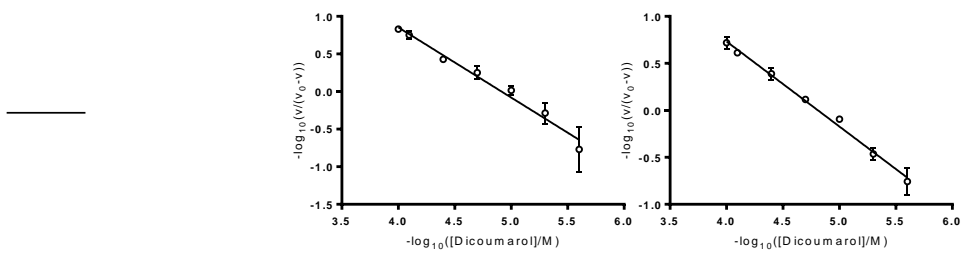

Figure 2. (A) Steady state kinetics for WT MdaB and variants: the dependence of rate on NADPH concentration, rates were measured at $37^{\circ} \mathrm{C}$ with $70 \mu \mathrm{M}$ DCPIP (dimer

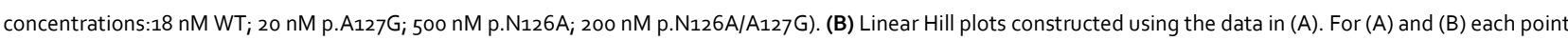
represents the mean of three separate determinations and the error bar the standard deviation of the mean. (C) Inhibition by nicotinamide carried out at $70 \mu \mathrm{M} N \mathrm{NDPH}$ and 70 $\mu \mathrm{M}$ DCPIP (D) Inhibition by NADP ${ }^{+}$carried out at $70 \mu \mathrm{M}$ NADPH and $70 \mu \mathrm{M}$ DCPIP (E) Inhibition by dicoumarol carried out at $150 \mu \mathrm{M}$ NADPH and $70 \mu \mathrm{M}$ DCPIP. Linear Hill plots in (C), (D) and (E) were constructed to estimate the Hill coefficient for each inhibitor. Each point represents the mean of three separate determinations and the error bars show the standard deviation of this mean. This was repeated three times (one representative Hill plot shown corresponding to the median value for $h$ ). 


\section{WT}
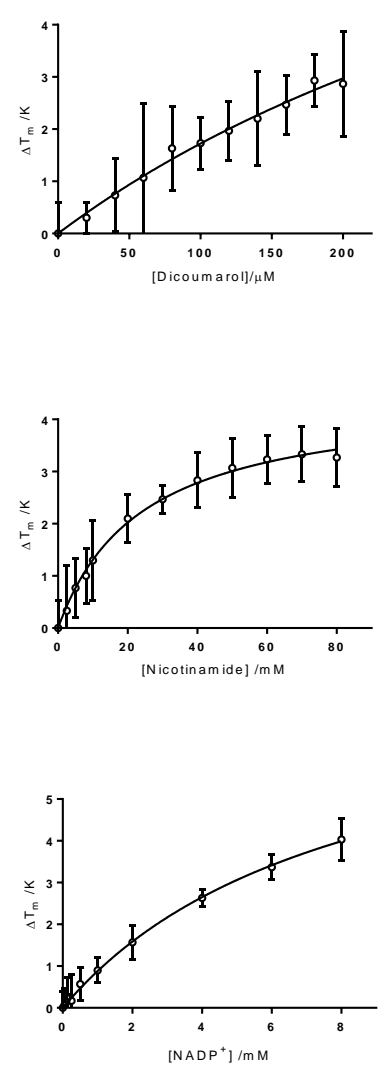

A127G
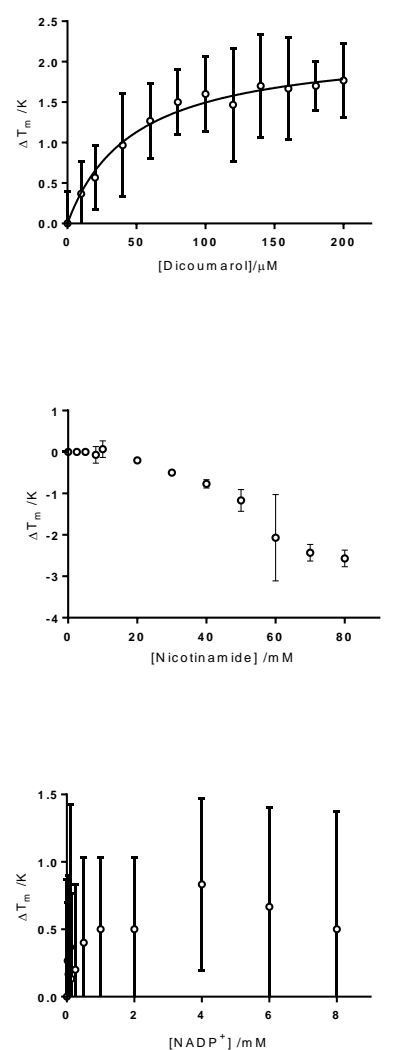

N126A

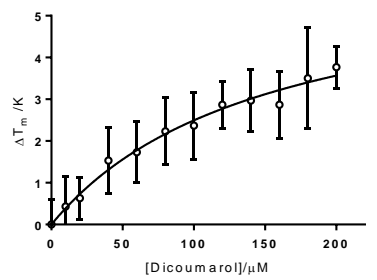

(1)

\section{N126A/A127G}

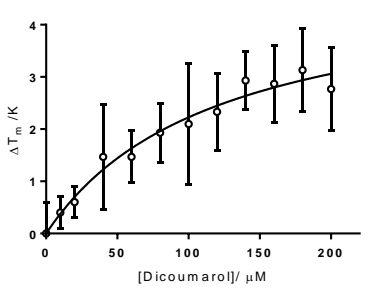

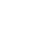

Figure 3. Dependence of thermal stability on ligand concentration; $T_{m}$ values determined from first derivative curves and plotted against the corresponding ligand concentration. Each point represents the mean of three separate determinations and the error bars were calculated from the standard deviations of these means. Dicoumarol

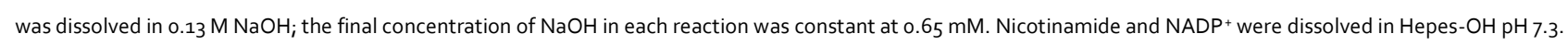




\section{HELVETICA}

\section{Conclusions}

MdaB has been characterized in terms of stability and oxidoreductase activity. Negative cooperativity and slight positive cooperativity towards $\mathrm{NADP}^{+}$and nicotinamide respectively, occur in wild-type MdaB. The change from alanine to glycine did not change the cooperative behaviour of the enzyme. The reduction in steric hindrance afforded by the change from asparagine to alanine resulted in enhanced inhibition by dicoumarol. Dicoumarol did not induce negative cooperativity in this variant. The introduction of both changes simultaneously did not allow the enzyme to exhibit negative cooperativity towards dicoumarol. A communication pathway linking the active sites through changes in conformation and mobility has been computationally identified in human $\mathrm{NOO}_{1}{ }^{[25]}$. Structural alignment of $\mathrm{MdaB}$ with human $\mathrm{NQO}_{1}$ indicates the presence of an equivalent structure in MdaB. However, there are no residues within this pathway which match those in the pathway in NQO1. Despite this, the bacterial enzyme exhibits negative cooperativity towards the product, $\mathrm{NADP}^{+}$. This suggests that the observed negative cooperativity propagates through a different pathway or that the communication occurs through the same connecting pathway as that in $\mathrm{NOO}_{1}$, but by a different mechanism. MdaB's ability to exhibit negative cooperativity towards $\mathrm{NADP}^{+}$while also exhibiting Michaelis-Menten behaviour towards NADPH may have evolved to confer the ability to regulate homeostasis within the quinone cycles. Negative cooperativity in the inhibition by NADP ${ }^{+}$allows for a greater sensitivity over a wider concentration range ${ }^{[33]}$ and therefore tune the enzyme's response to the supply of quinone from the reaction catalysed by QuMo. MdaB's tolerance to alteration of asparagine-127 to alanine in the active site suggests that additional changes to increase active site volumes would further broaden the range of molecules which bind to the enzyme. This would be useful for the generation of variants of the enzyme for use in biocatalysis.

\section{Experimental Section}

\section{Enzyme expression, purification and crosslinking:}

The coding sequence for MdaB was amplified by PCR using genomic DNA from $E$. coli $\mathrm{HMS}_{174}\left(\mathrm{DE}_{3}\right)$ as template. The $\mathrm{PCR}$ product was inserted into the pET-46EK/LIC vector (Merck, UK) as described in the manufacturer's instructions. Site-directed mutagenesis using the QuikChange protocol [34] was used to make changes in the coding sequence. Sequences were verified by DNA sequencing (GATC, London, UK;). The vector inserts a sequence which codes for residues, MAHHHHHHVDDDDK at the $5^{\prime}$ end of the gene enabling purification of the recombinant proteins by $\mathrm{Ni}^{2+}$ chromatography (His-Select, Sigma, UK); expression and purification were carried out as previously described for budding yeast Lot6p ${ }^{[20]}$. Protein concentrations were estimated by the method of Bradford using BSA as a control [35] Chemical crosslinking was carried out as previously described [20].

\section{Enzyme kinetics}

MdaB activity was measured at $37^{\circ} \mathrm{C}$ in either $50 \mathrm{mM}$ phosphate buffer $\mathrm{pH}$ 7.4 or $50 \mathrm{mM}$ HEPES-OH buffer $\mathrm{pH} 7.33$ using NADPH as the electron donor and DCPIP as the electron acceptor; lysozyme was included as a crowding agent (final concentration, $0.9 \mu \mathrm{M}$ ). Initial rates were determined by measuring the rate of decrease in $A_{600}$ resulting from the reduction of DCPIP. Readings were taken every $5 \mathrm{~s}$ and all rates were obtained from the linear section at the beginning of each progress curve. All reactions were carried out in triplicate in 96-well plates. Rates were measured at increasing concentrations of NADPH and a constant concentration of DCPIP $(70 \mu \mathrm{M})$. Corresponding background rates for the non-enzymatic reduction of DCPIP $(70 \mu \mathrm{M})$ by NADPH at each concentration (at $37^{\circ} \mathrm{C}$ ) were subtracted to give enzyme-catalysed rates. A calibration curve of $A_{600}$ against [DCPIP] was constructed in triplicate (in both buffers) and the resulting equation of the line was used to determine $\varepsilon L$ in the Beer-Lambert equation $(A=\varepsilon L C)$ this was used to convert all rates from rate of change in $A_{60}$ to rates in terms of change in [DCPIP]. The enzyme-catalysed rates were then divided by the [Enzyme]; the apparent Michaelis constant $\left(K_{\mathrm{m}, \mathrm{app}}\right)$ and turnover number $\left(k_{\mathrm{cat}, \mathrm{app}}\right)$ were determined from plots of enzyme-catalysed rate divided by [Enzyme] against the corresponding [NADPH]. The data were fitted to equation (1) using nonlinear curve fitting in GraphPad Prism 6.o (GraphPad Software Inc. CA., USA).

$\mathrm{v} /[\mathrm{E}]=k_{\mathrm{cat}, \mathrm{app}}[\mathrm{NADPH}] /\left(K_{\mathrm{m}, \mathrm{app}}+[\mathrm{NADPH}]\right)$

Where $k_{\mathrm{cat}, \text { app }}$ is the apparent turnover number, $K_{\mathrm{m} \text {,app }}$ is the apparent Michaelis constant and $[E]$ is the concentration of enzyme. These data were also used to construct linearised Hill plots of $-\log _{10}\left(\left(\mathrm{v} /[\mathrm{E}] / k_{\mathrm{cat}, \text { app }}{ }^{-}\right.\right.$ $(v /[E])))$ against $\log _{10}([N A D P H])$ and a value for the Hill coefficient (the gradient) obtained ${ }^{[36]}$.

Potential inhibitors (resveratrol, dicoumarol, AMP, NADP+, nicotinamide, folic acid, nicotinic acid and curcumin were tested. With the exception of nicotinamide and $\mathrm{NADP}^{+}$, none of these inhibited. Nicotinamide (0-300 $\mathrm{mM})$ and $\mathrm{NADP}^{+}(0-8 \mathrm{mM})$ were added into the reaction catalysed by WT $\mathrm{MdaB}$ and the variant p.A127G and their effect on the rate measured at 40 $\mu \mathrm{M}$ and $70 \mu \mathrm{M}$ NADPH and constant [DCPIP] (70 $\mu \mathrm{M})$. Dixon plots ${ }^{[37]}$ were constructed for each concentration of NADPH and the apparent competitive inhibition constant $\left(K_{\mathrm{ic}, \text { app }}\right)$ obtained as the intersection point of the lines. [S]/v plots ${ }^{\left[{ }^{8}\right]}$ were also constructed and where mixed inhibition was observed, the apparent uncompetitive inhibition constant $\left(K_{\mathrm{iu}, \mathrm{app}}\right)$ determined as the intersection point of the lines. Dicoumarol (o$100 \mu \mathrm{M}$ ) was tested as an inhibitor of WT MdaB, p.A127G and p.N126A. Dicoumarol was dissolved in $0.13 \mathrm{M} \mathrm{NaOH}$ and the concentration of $\mathrm{NaOH}$ was constant $(0.65 \mathrm{mM})$ in all reactions including those with zero dicoumarol. The initial rates of inhibited reactions were carried out at constant concentrations of DCPIP and NADPH (70 $\mu \mathrm{M}$ each) for WT MdaB and p.A127G. Linearized Hill plots of $-\log _{10}\left(v /\left(v_{0}-v\right)\right)$ against $\log _{10}\left[\right.$ Inhibitor] (where $v$ is the rate of the inhibited reaction and $v_{0}$ is the uninhibited rate) were constructed; the Hill coefficient is the gradient of these plots. 


\section{HELVETICA}

\section{Differential scanning fluorimetry}

Differential scanning fluorimetry was carried out as previously described ${ }^{[20]}$. An initial titration was carried out to identify an enzyme concentration which gave an optimum fluorescent signal. Enzymes were diluted in 50 mM HEPES, pH 7.33 to final concentrations in the range $0.25 \mu \mathrm{M}$ to $5 \mu \mathrm{M}$ dimer. This assay was also used to obtain apparent binding constants for inhibitors. The melting temperature of enzyme at each inhibitor concentration was plotted against the corresponding concentration of inhibitor and the data were fitted to equation (3) using non-linear curve fitting in GraphPad Prism.

$\Delta T_{m}=\left(\Delta T_{m, \max }[\right.$ Ligand $\left.]\right) /\left(K_{D, a p p}+[\right.$ Ligand $\left.]\right)$

Where $\Delta T_{m, \text { max }}$ is the maximum, limiting change in $T_{m}$ and $K_{\mathrm{D}, \text { app }}$ is the apparent dissociation constant for ligand and protein.

\section{Supplementary Material}

Supporting information for this article is available on the WWW under http://dx.doi.org/10.1002/MS-number.

\section{Acknowledgements}

CFM thanks the Department of Employment and Learning, Northern Ireland (DELNI, UK) for a PhD studentship. We thank Prof Aaron Maule (IGFS, QUB) for access to a qPCR machine used in the DSF assays.

\section{Author Contribution Statement}

CFM conducted all experiments and analyses. DJT conceived the project and supervised CFM. DJT and CFM co-wrote the manuscript.

\section{References}

[1] A. T. Dinkova-Kostova, P. Talalay, 'NAD(P)H:quinone acceptor oxidoreductase 1 (NOO1), a multifunctional antioxidant enzyme and exceptionally versatile cytoprotector', Archi Biochem Biophys 2010, 501, 116-123.

[2] S. Chen, K. Wu, R. Knox, 'Structure-function studies of DTdiaphorase (NOO1) and $\mathrm{NRH}$ : quinone oxidoreductase (NOO2)', Free Radic Biol Med 2000, 29, 276-284.

[3] S. K. Beaver, N. Mesa-Torres, A. L. Pey, D. J. Timson, 'NOO1: A target for the treatment of cancer and neurological diseases, and a model to understand loss of function disease mechanisms', Biochim Biophys Acta 2019, 1867, 663-676.

[4] D. Ross, J. K. Kepa, S. L. Winski, H. D. Beall, A. Anwar, D. Siegel, 'NAD(P)H:quinone oxidoreductase 1 (NOO1): chemoprotection, bioactivation, gene regulation and genetic polymorphisms', Chem Biol Interact 2000, 129, 77-97.

[5] M. A. Adams, P. Iannuzzi, Z. Jia, 'MdaB from Escherichia coli: cloning, purification, crystallization and preliminary $X$-ray analysis', Acta Crystallogr $F$ 2005, 61, 235-238.

[6] L. K. Green, A. C. La Flamme, D. F. Ackerley, 'Pseudomonas aeruginosa $\mathrm{MdaB}$ and $\mathrm{WrbA}$ are water-soluble two-electron quinone oxidoreductases with the potential to defend against oxidative stress', J Microbiol 2014, 52, 771-777.

[7] S. Zenno, H. Koike, A. N. Kumar, R. Jayaraman, M. Tanokura, K. Saigo, 'Biochemical characterization of NfsA, the Escherichia coli major nitroreductase exhibiting a high amino acid sequence homology to Frp, a Vibrio harveyi flavin oxidoreductase', J Bacteriol 1996, 178, 4508-4514.
[8] S. Zenno, H. Koike, M. Tanokura, K. Saigo, 'Gene cloning, purification, and characterization of $\mathrm{NfsB}$, a minor oxygeninsensitive nitroreductase from Escherichia coli, similar in biochemical properties to FRase I, the major flavin reductase in Vibrio fischeri', J Biochem 1996, 120, 736-744.

[9] J. W. Choi, J. Lee, K. Nishi, Y. S. Kim, C. H. Jung, J. S. Kim, 'Crystal structure of a minimal nitroreductase, ydjA, from Escherichia coli K12 with and without FMN cofactor', J Mol Biol 2008, 377, 258-267.

[10] G. A. Prosser, J. N. Copp, S. P. Syddall, E. M. Williams, J. B. Smaill, W. R. Wilson, A. V. Patterson, D. F. Ackerley, 'Discovery and evaluation of Escherichia coli nitroreductases that activate the anti-cancer prodrug CB1954', Biochem Pharmacol 2010, 79, 678-687.

[11] M. A. Adams, Z. Jia, 'Modulator of drug activity B from Escherichia coli: crystal structure of a prokaryotic homologue of DT-diaphorase', J Mol Biol 2006, 359, 455-465.

[12] M. A. Adams, Z. Jia, 'Structural and biochemical evidence for an enzymatic quinone redox cycle in Escherichia coli: identification of a novel quinol monooxygenase', J Biol Chem 2005, 280, 83588363 .

[13] S. Jemli, D. Ayadi-Zouari, H. B. Hlima, S. Bejar, 'Biocatalysts: application and engineering for industrial purposes', Crit Rev Biotechnol 2016, 36, 246-258.

[14] S. Hosoda, W. Nakamura, K. Hayashi, 'Properties and reaction mechanism of DT diaphorase from rat liver', J Biol Chem 1974, $249,6416-6423$.

[15] S. Sollner, R. Nebauer, H. Ehammer, A. Prem, S. Deller, B. A. Palfey, G. Daum, P. Macheroux, 'Lot6p from Saccharomyces cerevisiae is a FMN-dependent reductase with a potential role in quinone detoxification', FEBS $J$ 2007, 274, 1328-1339.

[16] A. L. Lovering, E. I. Hyde, P. F. Searle, S. A. White, 'The structure of Escherichia coli nitroreductase complexed with nicotinic acid: three crystal forms at 1.7 A, 1.8 A and 2.4 A resolution', J Mol Biol 2001, 309, 203-213.

[17] Y. Yanto, M. Hall, A. S. Bommarius, 'Nitroreductase from Salmonella typhimurium: characterization and catalytic activity', Org Biomol Chem 2010, 8, 1826-1832.

[18] Y. Hong, G. Wang, R. J. Maier, 'The NADPH quinone reductase MdaB confers oxidative stress resistance to Helicobacter hepaticus', Microb Pathog 2008, 44, 169-174.

[19] C. F. Megarity, J. R. Gill, M. C. Caraher, I. J. Stratford, K. A. Nolan, D. J. Timson, 'The two common polymorphic forms of human NRH-quinone oxidoreductase $2\left(\mathrm{NOO}_{2}\right)$ have different biochemical properties', FEBS Letts 2014, 588, 1666-1672.

[20] C. F. Megarity, H. K. Looi, D. J. Timson, 'The Saccharomyces cerevisiae quinone oxidoreductase Lot6p: stability, inhibition and cooperativity', FEMS Yeast Res 2014, 14, 797-807.

[21] B. Rase, T. Bartfai, L. Ernster, 'Purification of DT-diaphorase by affinity chromatography. Occurrence of two subunits and nonlinear Dixon and Scatchard plots of the inhibition by anticoagulants', Arch Biochem Biophys 1976, 172, 380-386.

[22] A. L. Pey, C. F. Megarity, D. J. Timson, 'NAD(P)H quinone oxidoreductase (NOO1): an enzyme which needs just enough mobility, in just the right places', Biosci Rep 2019, 39, BSR20180459.

[23] K. Gunasekaran, B. Ma, R. Nussinov, 'Is allostery an intrinsic property of all dynamic proteins?', Proteins 2004, 57, 433-443.

[24] N. M. Goodey, S. J. Benkovic, 'Allosteric regulation and catalysis emerge via a common route', Nat Chem Biol 2008, 4, 474-482.

[25] C. F. Megarity, H. A. Bettley, M. C. Caraher, K. A. Scott, R. C. Whitehead, T. A. Jowitt, A. Gutierrez, R. A. Bryce, I. J. Stratford, K. A. Nolan, D. J. Timson, 'Negative cooperativity in NAD(P)H quinone oxidoreductase $1\left(\mathrm{NOO}_{1}\right)^{\prime}$, ChemBioChem 2019, In press.

[26] G. Asher, O. Dym, P. Tsvetkov, J. Adler, Y. Shaul, 'The crystal structure of $\mathrm{NAD}(\mathrm{P}) \mathrm{H}$ quinone oxidoreductase 1 in complex with its potent inhibitor dicoumarol', Biochemistry 2006, 45, $6372-6378$

[27] F. Huang, W. M. Nau, 'A conformational flexibility scale for amino acids in peptides', Angew Chemie Int Ed 2003, 42, 22692272. 


\section{HELVETICA}

[28] A. Fersht, Structure and Mechanism in Protein Science: A Guide to Enzyme Catalysis and Protein Folding, W. H. Freeman, 1999

[29] M. Hayashi, K. Hasegawa, Y. Oguni, T. Unemoto,

'Characterization of FMN-dependent NADH-quinone reductase induced by menadione in Escherichia coli', Biochim Biophys Acta 1990, 1035, 230-236.

[30] A. Levitzki, D. E. Koshland, Jr., 'Negative cooperativity in regulatory enzymes', Proc Natl Acad Sci USA 1969, 62, 11211128.

[31] R. A. Cook, D. E. Koshland, Jr., 'Positive and negative cooperativity in yeast glyceraldehyde 3 -phosphate dehydrogenase', Biochemistry 1970, 9, 3337-3342.

[32] A. Cooper, M. A. Nutley, A. Wadood, in Protein-ligand interactions: A practical approach (Eds.: S. E. Harding, B. Chowdhury), Oxford University Press, Oxford, 2001.

[33] J. E. Ferrell, Jr., 'O\&A: Cooperativity', J Biol 2009, 8, 53.

[34] W. Wang, B. A. Malcolm, 'Two-stage PCR protocol allowing introduction of multiple mutations, deletions and insertions using QuikChange Site-Directed Mutagenesis', BioTechniques 1999, 26, 680-682.

[35] M. M. Bradford, 'A rapid and sensitive method for the quantitation of microgram quantities of protein utilizing the principle of protein-dye binding', Anal Biochem 1976, 72, 248254.

[36] D. J. Timson, 'Quantitative enzymology', Curr Enzym Inhib 2015, 11, 12-31.

[37] M. Dixon, 'The determination of enzyme inhibitor constants', Biochemical J 1953, 55, 170-171.

[38] A. Cornish-Bowden, 'A simple graphical method for determining the inhibition constants of mixed, uncompetitive and non-competitive inhibitors', Biochem J 1974, 137, 143-144. 
Entry for the Table of Contents

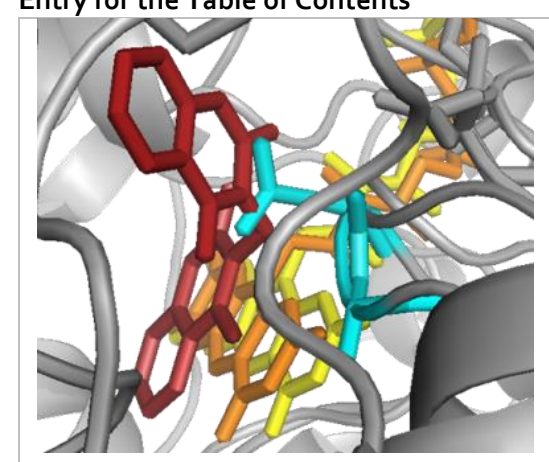

\section{Twitter}

Bacterial MdaB looks and acts like mammalian quinone oxidoreductases, but key details of its enzymology differ 